\title{
Practice and Knowledge of Funduscopy by Medical Practitioners in Owo, Nigeria
}

\section{CO Omolase FWACS, FMCOphth}

Department of Ophthalmology, Federal Medical Centre Owo, Ondo State, Nigeria

\section{SUMMARY}

Aim: This study was carried out to determine the practice of funduscopy by medical practitioners and also identify possible barriers to it in a Nigerian community.

Methodology: The study was conducted in March and April 2008 at the Federal Medical Centre, Owo, Ondo State, Nigeria. Eighty medical practitioners were selected by simple random sampling and interviewed with the aid of a questionnaire. Information obtained included biodata of the respondents, practice of funduscopy, barriers to the practice of funduscopy, as well as the use of dilating eye drops for funduscopy.

Results: More than half of the respondents (58.8\%) practiced funduscopy while the remaining $41.3 \%$ did not. Most respondents (89.4\%) who practiced funduscopy did so rarely. The majority of the respondents $(88.8 \%$ ) expressed a desire for further training in funduscopy. The barriers to the practice of funduscopy were mainly lack of skills (30\%) and lack of access to an ophthalmoscope $(28.8 \%)$. Very few respondents (7.5\%) used topical mydriatics for funduscopy. The level of experience and of the respondents significantly affected the practice of funduscopy with a $p$ value of 0.032 .

Conclusion: More than half of the respondents performed funduscopy, however most of them did so rarely. The barriers to the practice of funduscopy include lack of skills and access to an ophthalmoscope.

Key words: funduscopy, medical practitioners, direct ophthalmoscope.

\section{INTRODUCTION}

The ophthalmoscope was invented by Von Hemhotz in 1852. ${ }^{1}$ Direct ophthalmoscopy allows for visual examination of the retina and ocular media. The hand-held direct ophthalmoscope uses the patient's eye as a simple magnifier by aligning its viewing and illuminating beams. This produces an erect magnified, well-detailed real image of the retina. Compared to other fundus viewing instruments, the direct ophthalmoscope is easier to master, patients find it more comfortable, and it can be used to view smaller pupils. $^{2}$

Direct ophthalmoscopy is indicated for ocular fundus examination, evaluation of an eye's media and assessment of a patient's fixation pattern. ${ }^{2}$ The ophthalmoscope head connects to a handle that serves as the power source. The head of the ophthalmoscope contains a variable range of plus and minus lenses used to compensate for refractive errors. The light is projected through an adjustable aperture and correcting lens to illuminate the fundus. Examination of the ocular fundus is an integral part of physical examination in ophthalmology. ${ }^{3}$ Inspection of the fundus may help in the management of a number of systemic disorders such as diabetes mellitus and systemic lupus erythromatosus. ${ }^{4}$ Funduscopy is especially important in older patients in whom visual problems are common.$^{5}$ A study carried out in Liverpool highlighted the prevalence of reversible visual impairment in elderly patients admitted to hospital; thus reinforcing the importance of routine eye examination in patients. ${ }^{6}$

Although it is accepted that direct ophthalmoscopy is not a useful screening tool for detecting peripheral retinal diseases, ${ }^{7-11}$ ophthalmoscopy is the method of choice for nonophthalmologists when carrying out an examination of the fundus. Pharmacological pupillary dilatation using topical short-acting mydriatics like tropicamide, enhances the view of the fundus especially for non ophthalmologists performing funduscopy. There is a low potential risk of acute angle closure glaucoma, ${ }_{1}^{12}$ and toxicity, ${ }_{13}^{13}$ but these are rare and should not justify non-usage of topical mydriatics in routine practice of funduscopy.

It is important for medical practitioners to carry out funduscopy as part of the examination of the patient. There is, however, need for proper training of medical practitioners in funduscopy so that they can derive useful information from using the ophthalmoscope. The need for continuous medical education concerning funduscopy was stressed by Jackson et al., ${ }^{14}$ as well as Codiero and colleagues. ${ }^{15}$ It is common practice for the doctors in other departments in the

*Correspondence: Dr Charles O. Omolase, Department of Ophthalmology, Federal Medical Centre, PMB 1053, Owo, Ondo State, Nigeria E-mail: omolash2000@yahoo.com 
hospital to invite an ophthalmologist to perform the funduscopy, even before they have made an attempt. This study was designed to assess the practice of funduscopy by medical practitioners at Federal Medical Centre, Owo, Ondo State, Nigeria. Possible barriers to the practice of funduscopy were also identified. It is hoped that relevant policy implications drawn from this study will improve the practice of funduscopy.

\section{METHODOLOGY}

This study was conducted in March and April, 2008 at the Federal Medical Centre Owo, Ondo State, Nigeria. Owo is a rural community located in Ondo State, southwestern Nigeria. Informed consent was obtained from each of the respondents. Eighty medical doctors out of the one hundred and thirty-two practicing in the hospital were selected using simple random sampling and interviewed by the authors with the aid of a structured questionnaire. Information obtained from the respondents, with the aid of the study instrument (questionnaire), included their biodata, practice of funduscopy and how often they practiced funduscopy. The barriers to the practice of funduscopy were also obtained. The use of dilating eye drops in carrying out funduscopy and possible reasons for not using it were also established. The data obtained with the study instrument were collated and analysed using SPSS 12.0.1. Cross tabulation of the status/level of experience of the respondents and the practice of funduscopy was done. Statistical significance was set at $p$ equals or less than 0.05 .

\section{RESULTS}

Eighty medical practitioners participated in this study comprising of 12 consultants, 9 resident doctors, 40 medical officers and 19 house officers. The ages of the respondents ranged between 25 and 50 years. The mean age of the respondents was 33 years.

The ethnicity of the respondents revealed that $69(86.3 \%)$ were Yoruba, $8(10 \%)$ were Ibo, while the other ethnic groups accounted for the remaining 3 (3.8\%). Seventy-one (88.8\%) of the respondents were Christian, while the remaining 9 $(11.3 \%)$ were Muslim. Forty-four (55\%) were single, while 36 (45\%) were married.

Performance of funduscopy. The majority of respondents, $47(58.8 \%)$ performed funduscopy, while the remaining 33 $(41.3 \%)$ did not. The frequency of practice of funduscopy among the respondents varied as only one practitioner $(2.1 \%)$ did it on a weekly basis, $42(89.4 \%)$ practiced it rarely and the remaining $4(8.5 \%)$ practiced it occasionally.
About 75 (93.8\%) respondents considered funduscopy an important part of a physical examination; 5 (6.3\%) considered it unimportant.

The majority of the respondents, 79 (98.8\%) felt that their funduscopy skills could be improved, only one doctor $(1.3 \%)$ did not feel so. Seventy-one of the respondents $(88.8 \%)$ expressed their desire for training in funduscopy, while the remaining $9(11.3 \%)$ declined training. As revealed in table 1 the main barriers to practice of funduscopy were lack of funduscopy skills (30\%) and lack of access to ophthalmoscope $(28.8 \%)$.

Table 1. Barriers to practice of funduscopy

\begin{tabular}{lcc}
\hline Barrier & Frequency & $\begin{array}{c}\text { Percentage } \\
(\%)\end{array}$ \\
\hline Time constraints & 8 & 10.0 \\
Lack of skill & 24 & 30 \\
$\begin{array}{l}\text { Lack of access to } \\
\text { ophthalmoscope }\end{array}$ & 23 & 28.8 \\
Low clinical priority & 18 & 22.2 \\
$\begin{array}{l}\text { Poor appreciation of its } \\
\text { importance }\end{array}$ & 7 & 8.8 \\
\hline Total & 80 & 100 \\
\hline
\end{tabular}

A few practitioners $(7.5 \%)$ used topical mydriatics agents like tropicamide for dilatation of the pupils for funduscopy, while the majority $(92.5 \%)$ did not. The reasons for not using mydriatics as shown in table 2, were lack of awareness (41.3\%) and unavailability of the mydriatic agents (38.7\%). The medical status of the respondents (see table 3) significantly affected the practice of funduscopy with a $p$ value of 0.032

Table 2. Reasons for not using dilating eye drops

\begin{tabular}{lcc}
\hline Reasons & Frequency & $\begin{array}{c}\text { Percentage } \\
(\%)\end{array}$ \\
\hline Lack of awareness & 31 & 41.9 \\
$\begin{array}{l}\text { Unavailability of dilating eye } \\
\text { drops }\end{array}$ & 28 & 37.8 \\
$\begin{array}{l}\text { Able to view fundus without } \\
\text { dilating }\end{array}$ & 4 & 5.4 \\
$\begin{array}{l}\text { Worried about side effects } \\
\text { Time constraint }\end{array}$ & 6 & 8.1 \\
\hline Total & 5 & 6.8 \\
\hline
\end{tabular}




\section{DISCUSSION}

The age range of the respondents $-25-50$ years - is in tandem with the active work force that they belong to. The fact that most of the respondents were Yoruba is also expected, in view of the fact that the study area is a Yoruba community. Most of the respondents were Christian. The sample included the entire hierarchy of medical practitioners namely consultants, resident doctors, medical officers and house officers. This is expected to reduce bias.

More than half of the respondents had practiced funduscopy, however, the majority rarely practice it on a regular basis. This finding, though disappointing, is expected, as doctors, due to different reasons, do not perform funduscopy as much as they should. Ophthalmoscopy is taught in the medical schools in Nigeria. However, doctors are likely to lose the acquired ophthalmoscopy skills if they do not practice it. Funduscopy is a very important part of the physical examination that could help in the management of some systemic conditions. The direct ophthalmoscope is a small portable instrument that one can learn to use within a short time; however, the medical practitioner must practice funduscopy regularly in order to master the technique. The increasing workload of doctors could lead to the abandonment of funduscopy, with a bias towards the seemingly more important examinations to the detriment of funduscopy. A study carried out in UK by Roberts et al. ${ }^{3}$ amongst 41 general practitioners revealed that only $44 \%$ of them were confident in the use of funduscopy.

Another study done by Moral et al. ${ }^{16}$ among paediatricians also revealed that most participants felt they were not competent to perform funduscopy. Most respondents expressed the desire for training in funduscopy, and a majority felt that their funduscopy skills could be improved. These findings are consistent with that of Robert et al, in UK in which $83 \%$ of the respondents felt they would benefit from more training, and $97 \%$ felt that their funduscopy skills could be improved. The desire for training in funduscopy expressed by most of the respondents brings to the fore the need for continuous medical education, especially in a subspeciality like ophthalmology, as there is the tendency to pay less attention to it. The main identified barriers to the practice of funduscopy can easily be overcome by the provision of more ophthalmoscopes and meeting the training needs of medical practitioners by giving them adequate training in funduscopy. Very few respondents in this study used dilating eye drops to aid funduscopy. The drug of choice for dilating the pupils is tropicamide. It is known that the wider the pupil, the wider the view of the fundus. Dilation of the pupils often comes in handy especially when there are opacities in the media that could preclude a good view of the fundus. The fact that some of the respondents expressed concern about the side effects of the dilating drops is understandable. However, some authors have suggested using a low concentration $(0.25 \%$ $0.5 \%$ ) of tropicamide has been shown to minimize the adverse effects, while still achieving adequate dilation of the pupils, ${ }^{17}$ but the efficacy has not been established in blacks. It is possible to achieve optimal dilation of the pupils within thirty minutes using tropicamide. There is need to create more awareness about dilating eye drops among nonophthalmologists so as to enhance the practice of funduscopy. The dilating eye drops should also be made available so that medical practitioners can have access to the dilating agents while carrying out funduscopy on patients. It is understandable that a higher proportion of resident doctors (table 3) practiced funduscopy since they are in training, they are likely to examine their patients thoroughly. Another factor that likely contributed to this finding is the fact that the resident doctors in a department such as family medicine, undergo a one-month training in ophthalmology. Only 5 per cent of the consultants interviewed practiced funduscopy. This, however, is not surprising as it may not be particularly relevant to their area of specialization. The fact that less than half of the house officers interviewed performed funduscopy brings up the need to train junior doctors to perform funduscopy. This can be addressed by introducing a two-week posting for all the house officers in ophthalmology.

Structured final examination that includes funduscopy for medical students should also be introduced.

Table 3. Cross tabulation of medical practitioners who practice of funduscopy hierarchy

\begin{tabular}{lccc}
\hline \multirow{2}{*}{$\begin{array}{c}\text { Level of } \\
\text { experience/hierarchy }\end{array}$} & \multicolumn{3}{c}{ Practice of Funduscopy } \\
\cline { 2 - 4 } & Yes & No & Total \\
\hline Consultant & 4 & 8 & 12 \\
Resident doctor & 7 & 2 & 9 \\
Medical officer & 28 & 12 & 40 \\
House officer & 8 & 11 & 19 \\
\hline Total & 47 & 33 & 80 \\
\hline
\end{tabular}

\section{CONCLUSION}

More than half of the respondents have practiced funduscopy; however, the majority of them practice it rarely. The main identified barriers to the practice of funduscopy were lack of skills in funduscopy and lack of access to the direct ophthalmoscope. Most respondents expressed the desire for training in funduscopy so as to improve their skills. The cadre of the respondents significantly affected the practice of funduscopy. 


\section{RECOMMENDATION}

There is need to pay more attention to training of medical students in the art of funduscopy during their posting in ophthalmology.

House officers should routinely rotate through ophthalmology for a period not less than two weeks during their one year internship to enable them acquire proficiency in funduscopy, which will be of use to them as medical practitioners.

The practice of funduscopy amongst medical practitioners should be encouraged and their training needs in respect of funduscopy should be met through continuous medical education.

Hospital authorities should make direct ophthalmoscopes and dilating eye drops readily available.

\section{ACKNOWLEDGEMENT}

Special thanks to the respondents for graciously accepting to participate in this study. The support of the management of the Federal Medical Centre, Owo, Ondo State, Nigeria is hereby acknowledged.

\section{REFERENCES}

1. Von Helmhotz H. Description of an ophthalmoscope for examining the retina of the living eye. AMA Arch Ophthalmol 1951; 46: 565-83.[Pubmed].

2. Roux P. Ophthalmoscopy for general practitioner. $S A$ Fam Pract 2004; 46(5):10-1.

3. Roberts E, Morgan R, King D and Cherkin L. Funduscopy a forgotten art? Post Grad Med J 1999; 75: 282-4.

4. Macleod J, Munro J. The use of the ophthalmoscope. In: Macleod J, Munro J, eds. Clinical examination.UK: Churchill Livingstone ,1986: 408-20.

5. Wormald RP, Wright LA, Courtney P, Beaumont B, Haines AP. Visual problems in the elderly population and implications for services .BMJ 1992; 304: 1226-9.

6. Jack CI, Smith T, Neoh C, Lye M, McGalliard JN. Prevalence of low vision in the elderly patients admitted to an acute geriatric unit in Liverpool: elderly people who fall are more likely to have low vision. Gerentology 1995; 41(5): 280-5.

7. Pollack AL, Brodie SE. Diagnostic yield of the routine dilated fundus examination .Ophthalmology 1998;105 (2): 382-6.

8. Batchelder TJ, Fireman B, Friedman GD et al. The value of routine dilated pupil screening examination .Arch Ophthalmol 1997; 115 (9): 1179-84.

9. Garvican L, Clowes J, Gillow T. Preservation of sight in diabetes: developing a national risk reduction programme. Diabet Med 2000; 17 (9): 627-34.

10. Hutchinson A, McIntosh A, Peters J et al. Effectiveness of screening and monitoring tests for diabetic retinopathy - a systematic review. Diabet Med 2000; 17 (7): 495-506.

11. Owens DR, Gibbins RC, Lewis PA, Wall S, Allen JC, Morton R. Screening for diabetic retinopathy by general practitioners: ophthalmoscopy or retinal photography as $35 \mathrm{~mm}$ colour transpariencies ? Diabet Med 1998; 15 (5): $170-5$.

12. Wolfs RC, Grobbee DE, Hofman A, de Jong PT. Risk of acute angle closure-glaucoma after diagnostic mydriasis in non selected subjects: the Rotterdam study .Invest Ophthalmol Vis Sci 1997; 38(12): 2683-7.

13. Brunner GA, Fleck S, Pieber TR et al. Near fatal anti cholinergic intoxication after routine funduscopy. Intensive Care Med 1998; 24(7): 730-1.

14. Jackson C, de Jong I, Glasson W. Royal Australian College of Ophthalmologists and Royal Australian College of General Practitioners National GP Eye skills Workshops: colleges and divisions re skilling general practice. Clin Experiment Ophthalmol 2000; 28: 347-9.

15. Cordeiro MF, Jolly BC, Dacre JE. The effect of formal instruction in ophthalmoscopy on medical students performance. Med Teach 1993; 15: 321-5.

16. Morad Y, Barkana Y, Avni I and Kozer E. Fundus anomalies: What the pediatrician's eye can't see. IJQHC 2004; 16(5): 363 -5.

17. Levine L. Mydriatic effectiveness of dilute combinations of phenylephrine and tropicamide. Am J Optom Phys Optics 1982; 59(7): 580 -94. 